\title{
A Uranium(VI)-Oxo-Imido Dimer Complex Derived from a Sterically Demanding Triamidoamine
}

Philip J. Cobb, Ashley J. Wooles, and Stephen T. Liddle*

Department of Chemistry, The University of Manchester, Oxford Road, Manchester, M13 9PL, UK.

*Email: steve.liddle@manchester.ac.uk

\section{Contents}

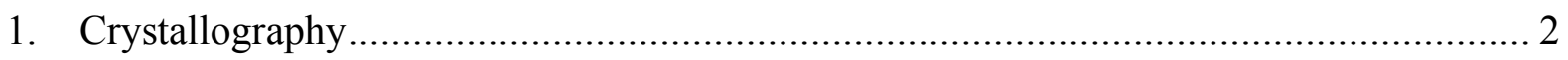

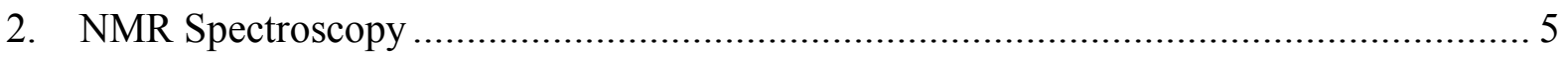

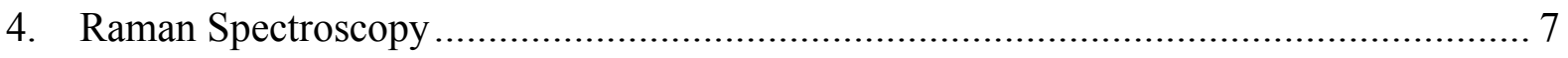

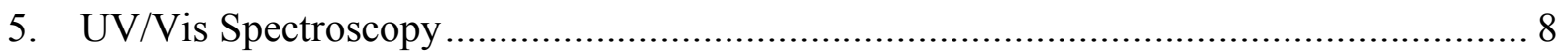

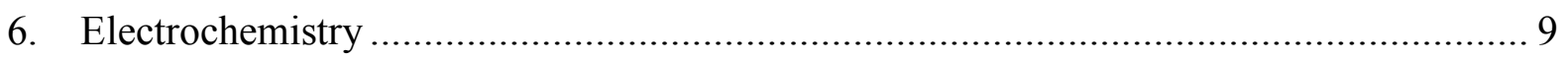

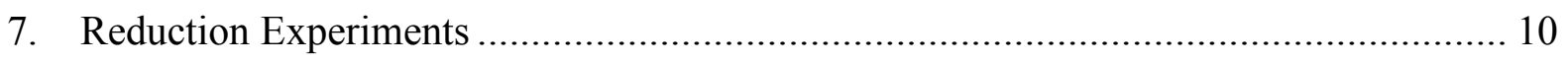

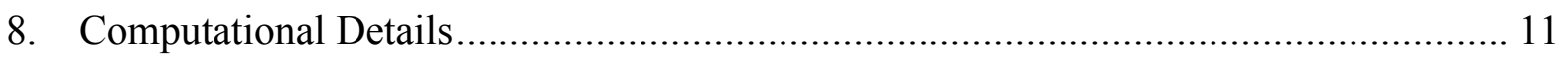

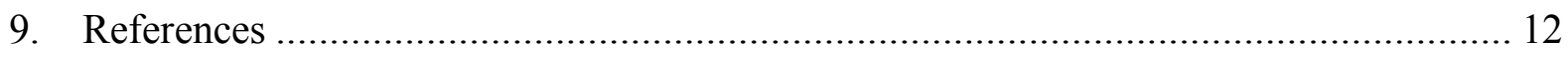




\section{Crystallography}

Crystallographic data was recorded on an Agilent SuperNova diffractometer utilising an Atlas CCD area detector and mirror-monochromated $\mathrm{CuK} \alpha$ radiation $(\lambda=1.54184)$. The intensities from the recorded data were integrated on $1^{\circ}$ frames by $\omega$ rotation. The cell parameters were refined from all the strong reflections observed in the data set. Gaussian face indexing methods were employed for absorption correction. Programs CrysAlisPro (control) and CrysAlisPro (integration) were used. ${ }^{1}$ The structures were solved on the program OLEX2 by either direct methods or charge flipping using SHELXS, SHELXT or superflip, and were refined by full-matrix least-squares on all unique $\mathrm{F}^{2}$ values using the ShelXL refinement package. ${ }^{2-5}$ All non-H atoms were refined with anisotropic displacement parameters. The largest residual electron density in the differential map was near to heavy atoms and of no chemical significance. Molecular graphics were produced using ORTEP-3 and POV-Ray. ${ }^{6,7}$

A summary of the general crystallographic data for complexes $\mathbf{1}$ and $\mathbf{2}$, are shown in Table 1. The complete crystallographic data for these complexes, including atom coordinates and displacement parameters, is available free of charge on the CCDC: 1954468-1954469, www.ccdc.cam.ac.uk/data_request/cif. 
Table S1. Crystallographic data for $\mathbf{1}$ and 2.

\begin{tabular}{|c|c|c|}
\hline Number & 1 & 2 \\
\hline Formula & $\mathrm{C}_{52} \mathrm{H}_{118} \mathrm{~N}_{8} \mathrm{O}_{3} \mathrm{Si}_{4} \mathrm{U}_{2}$ & $\mathrm{C}_{24} \mathrm{H}_{48} \mathrm{Cl}_{4} \mathrm{~K}_{2} \mathrm{Li}_{2} \mathrm{O}_{12}$ \\
\hline $\mathrm{Mr} / \mathrm{gmol}^{-1}$ & 1491.96 & 762.50 \\
\hline Cryst size /mm & $\begin{array}{l}0.125 \times 0.073 \times \\
0.026\end{array}$ & $0.1109 \times 0.0977 \times 0.0541$ \\
\hline Crystal system & monoclinic & monoclinic \\
\hline Space group & $P 2_{1} / n$ & $P 2{ }_{1} / c$ \\
\hline Radiation & $\mathrm{CuK} \alpha$ & $\mathrm{CuK} \alpha$ \\
\hline Collection Temp /K & $120(2)$ & $120(2)$ \\
\hline $\mathrm{a} / \AA$ & $8.6865(5)$ & $17.2106(5)$ \\
\hline $\mathrm{b} / \AA$ & $25.488(2)$ & $10.4044(3)$ \\
\hline $\mathrm{c} / \AA$ & $29.0738(13)$ & $20.0436(6)$ \\
\hline$\alpha /{ }^{\circ}$ & 90 & 90 \\
\hline$\beta /{ }^{\circ}$ & $98.668(6)$ & $91.508(2)$ \\
\hline$\gamma /{ }^{\circ}$ & 90 & 90 \\
\hline $\mathrm{V} / \AA^{3}$ & $6363.5(8)$ & $3587.87(18)$ \\
\hline $\mathrm{Z}$ & 4 & 4 \\
\hline$\rho_{\text {calc }} / \mathrm{gcm}^{-3}$ & 1.557 & 1.412 \\
\hline$\mu, \mathrm{mm}^{-1}$ & 15.270 & 5.531 \\
\hline No. of reflns measured & 45614 & 25553 \\
\hline No. of unique reflns, $\mathrm{R}_{\text {int }}$ & $11227,0.1761$ & $7142,0.0490$ \\
\hline $\begin{array}{l}\text { No. of reflns with } F^{2}> \\
2 \sigma\left(F^{2}\right)\end{array}$ & 7103 & 6240 \\
\hline $\begin{array}{l}\text { Transmission coefficient } \\
\text { range }\end{array}$ & $0.236-0.725$ & $0.286-0.570$ \\
\hline$R, R_{\mathrm{w}}\left(F^{2}>2 \sigma\left(F^{2}\right)\right)$ & $0.0847,0.2171$ & $0.0378,0.0993$ \\
\hline$R, R_{\mathrm{w}}$ (all data) & $0.1301,0.2658$ & $0.0439,0.1052$ \\
\hline$S$ & 1.069 & 1.031 \\
\hline Parameters, Restraints & 647,935 & 434,297 \\
\hline Max, min. diff map $/ \AA^{-3}$ & $3.80,-4.97$ & $0.349,-0.309$ \\
\hline
\end{tabular}




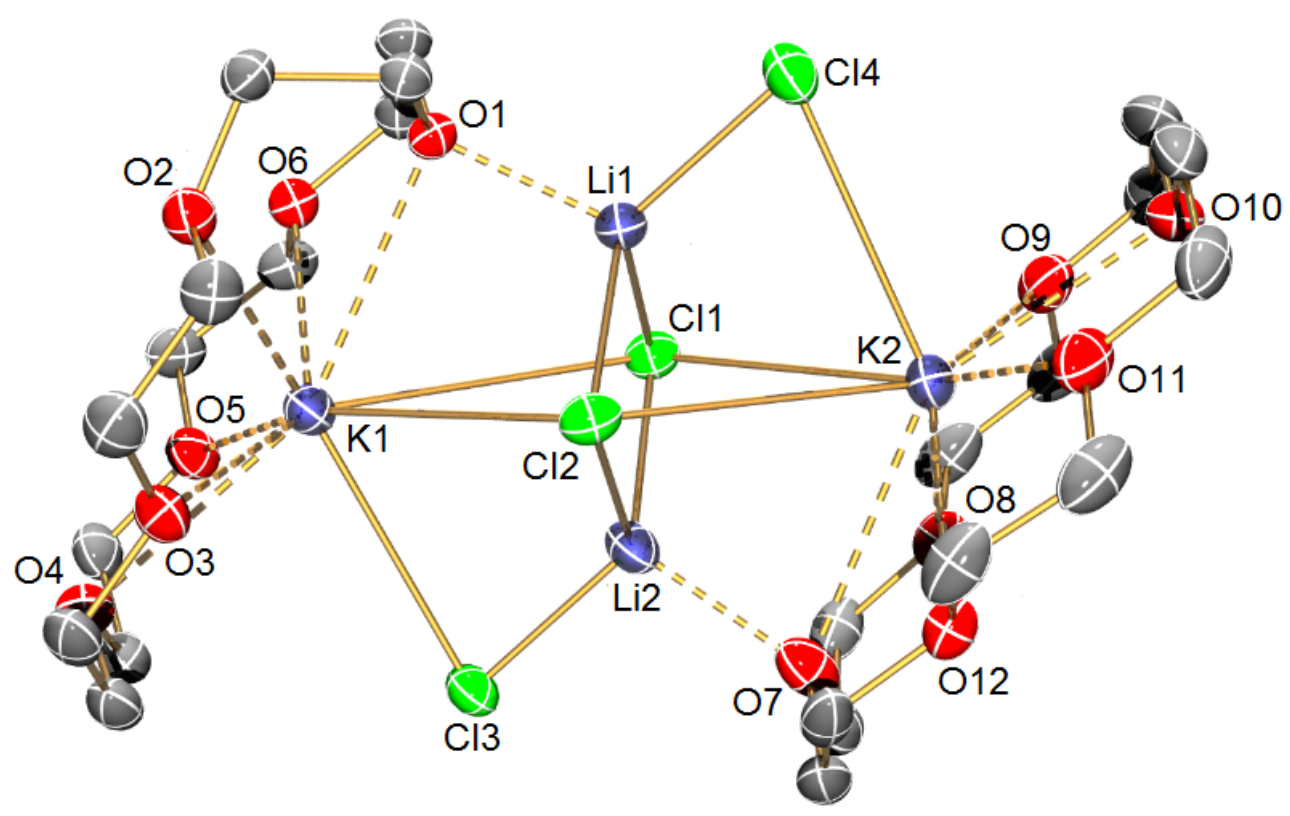

Figure S1. Solid state structure of 2 with selective labelling. Displacement ellipsoids set to $40 \%$, with hydrogen atoms and minor disorder components omitted for clarity. Selected bond lengths $(\AA)$ and angles $\left(^{\circ}\right)$ : K1-Cl1, 3.2785(6); K1-Cl2, 3.6487(6); K1-Cl3, 3.3588(6); K2C11, 3.7505(6); K2-Cl2, 3.3054(6); K2-Cl4, 3.2987(6); Li1-Cl1 2.361(4); Li1-Cl2, 2.385(3); Li1-Cl4, 2.226(3); Li2-Cl1， 2.331(3); Li2-Cl2，2.368(3); Li2-Cl3，2.265(3); K1-O1, 3.0805(15); K1-O2, 3.113(3); K1-O3, 2.7780(16); K1-O4, 2.8611(16); K1-O5, 2.7964(16); K1-O6, 2.9523(14); K2-O7, 2.9668(13); K2-O8, 2.9816(14); K2-O9, 2.7674(15); K2-O10, 2.9732(16); K2-O11, 2.7718(14); K2-O12, 2.9108(14); Li1-O1, 2.093(4); Li2-O7, 2.033(3); Cl1-K1-Cl2，59.691(12); Cl1-K2-Cl2，58.326(12)；Cl3-K1-O1， 132.47(3)；Cl4-K2-O7, 126.88(3); Cl1-Li1-Cl2, 93.67(12); Cl1-Li2-Cl2, 94.91(11); Li1-Cl4-K2, 74.41(9); Li2-Cl3K1, 70.89(8). 


\section{NMR Spectroscopy}

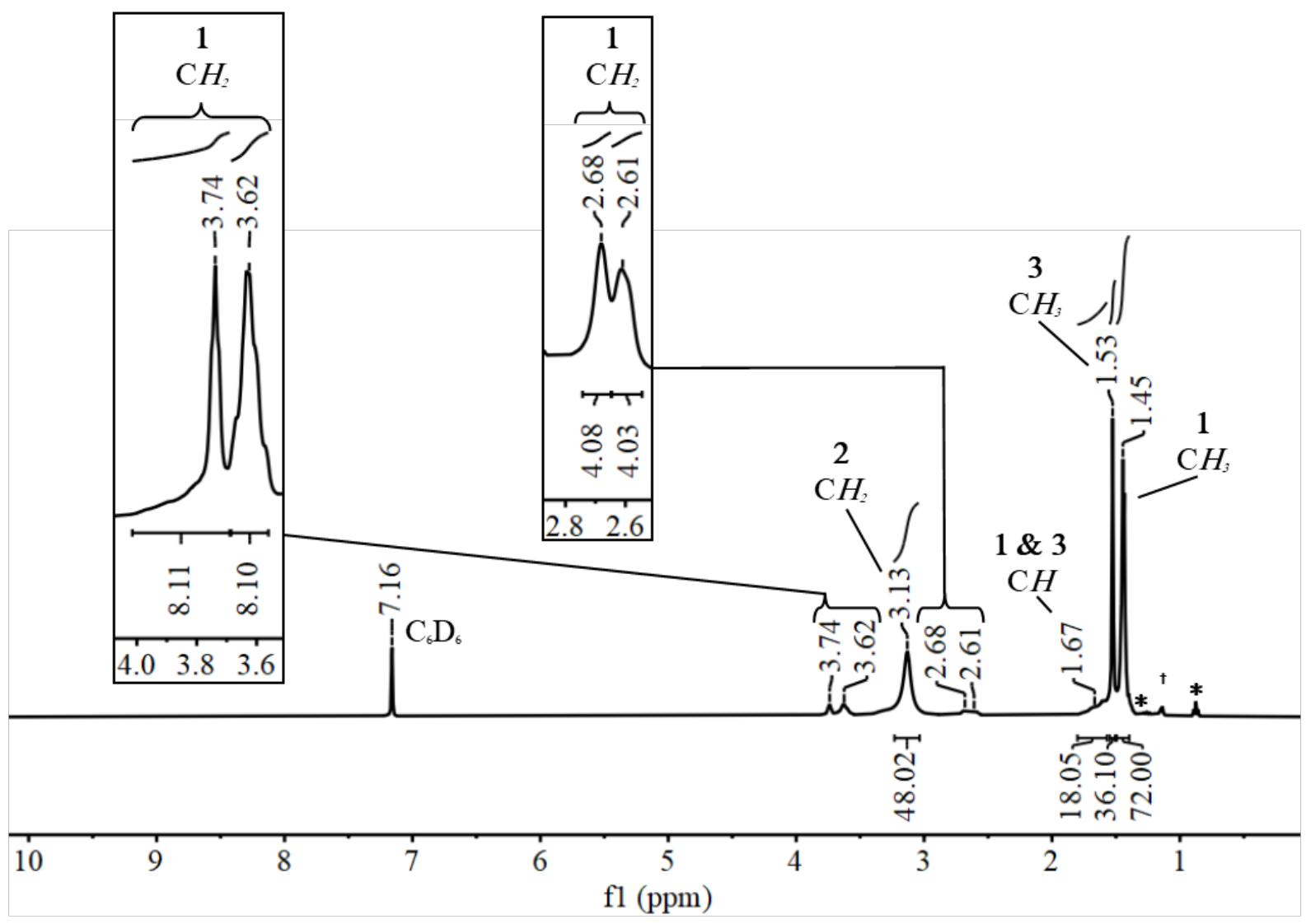

Figure S2. ${ }^{1} \mathrm{H}$ NMR of 1-3 $\left[*=\right.$ pentane, $\left.\dagger=\operatorname{Tren}{ }^{\mathrm{TIPS}} \mathrm{H}_{3}, \mathrm{Si}\left\{\mathrm{CH}\left(\mathrm{CH}_{3}\right)\right\}\right]$.

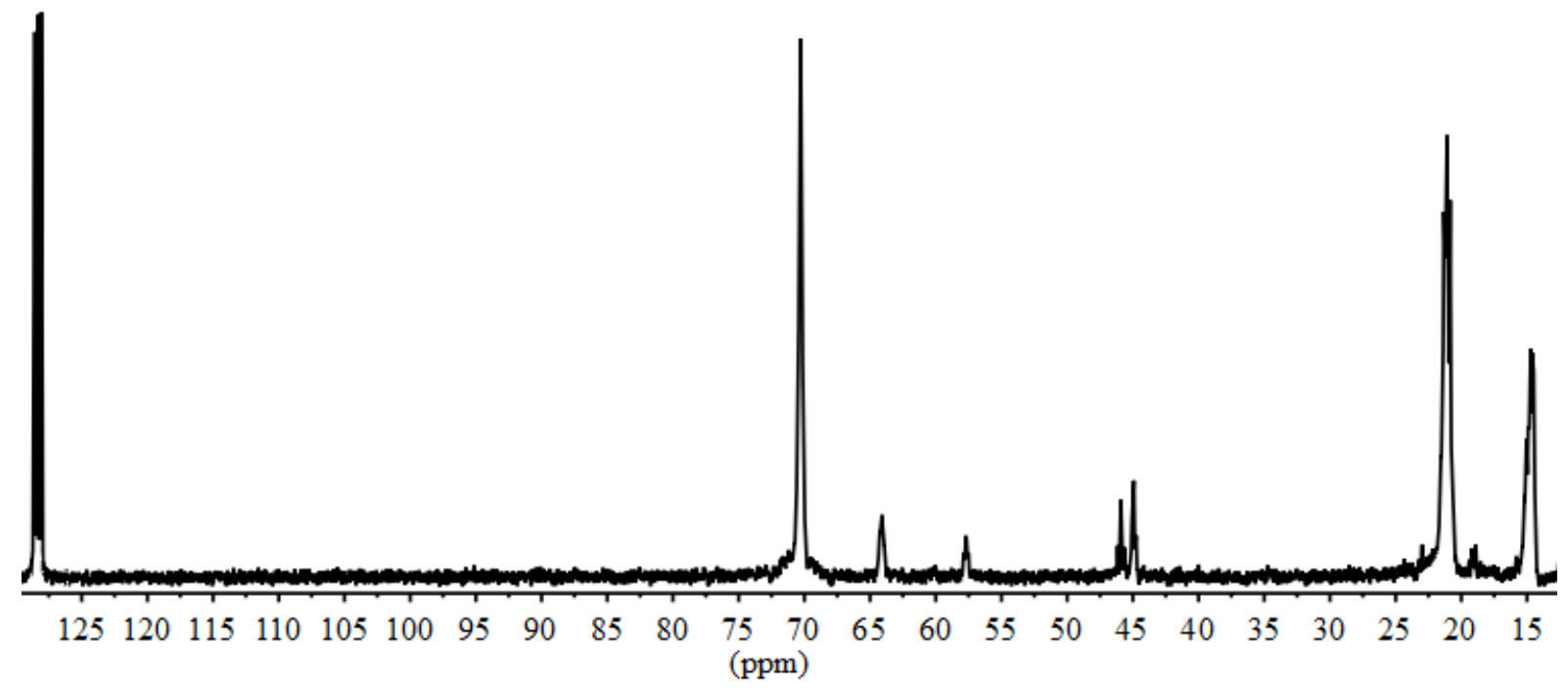

Figure S3. ${ }^{13} \mathrm{C}\left\{{ }^{1} \mathrm{H}\right\}$ NMR of 1-3. 


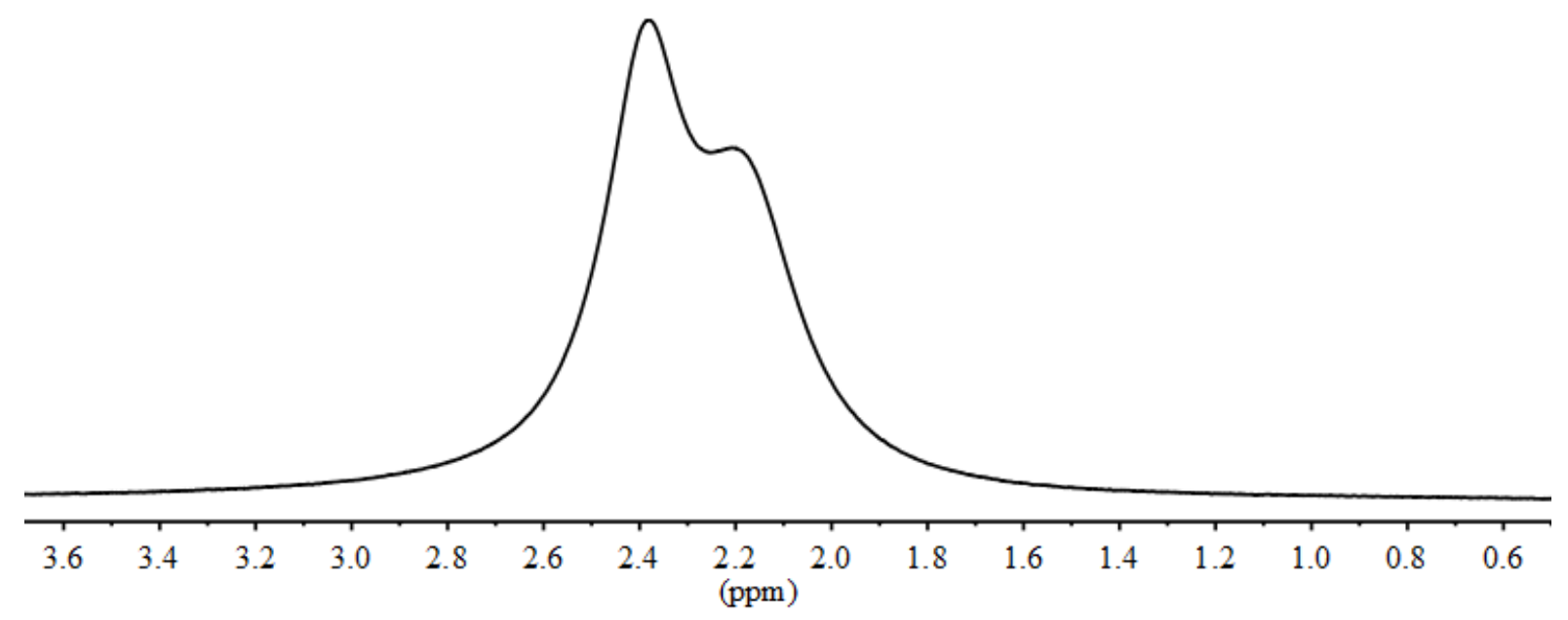

Figure S4. ${ }^{7} \mathrm{Li}\left\{{ }^{1} \mathrm{H}\right\}$ NMR of 2 and 3.

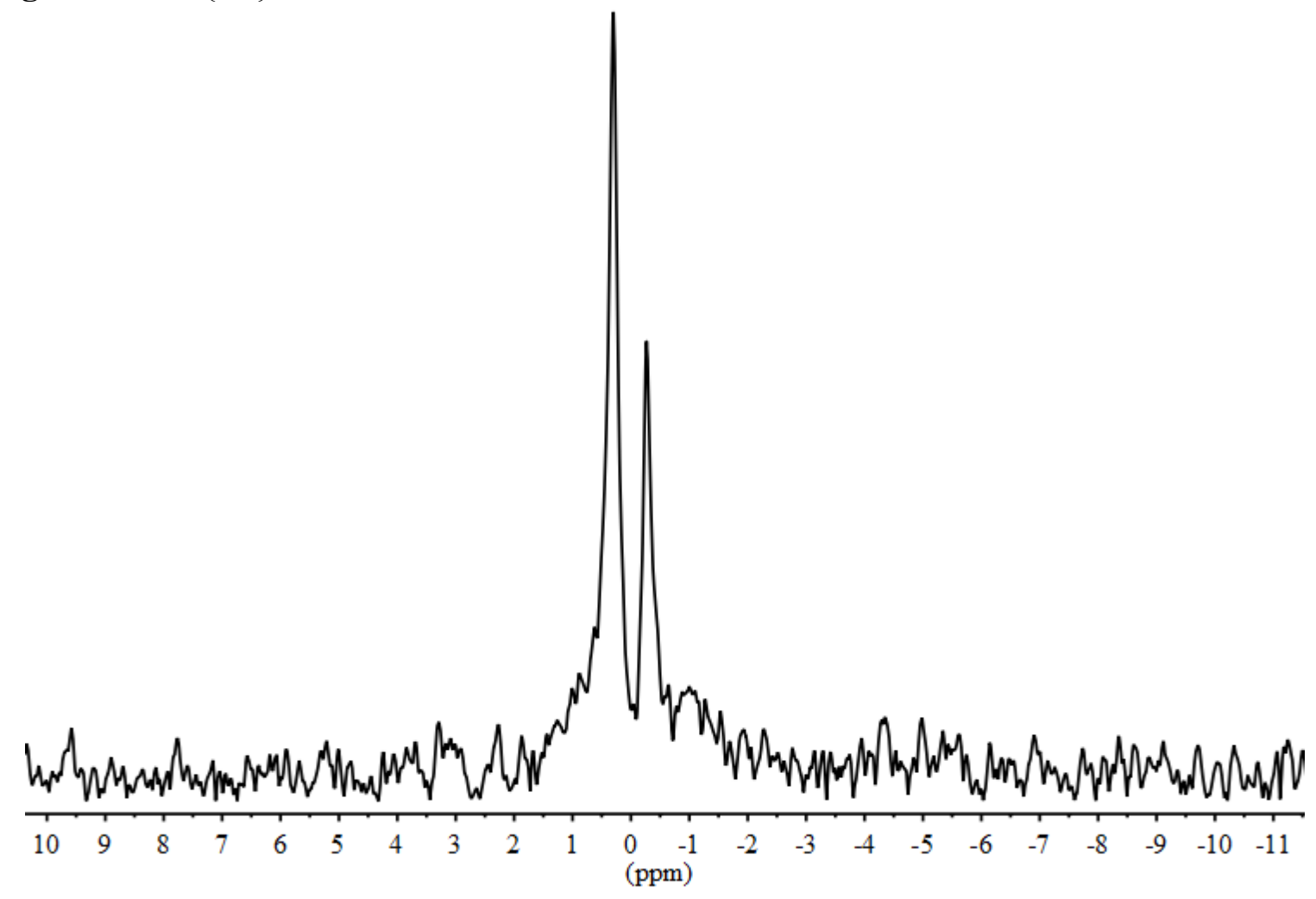

Figure S5. ${ }^{29} \mathrm{Si}\left\{{ }^{1} \mathrm{H}\right\}$ NMR of $\mathbf{1}$ and $\mathbf{3}$. 


\section{IR Spectroscopy}

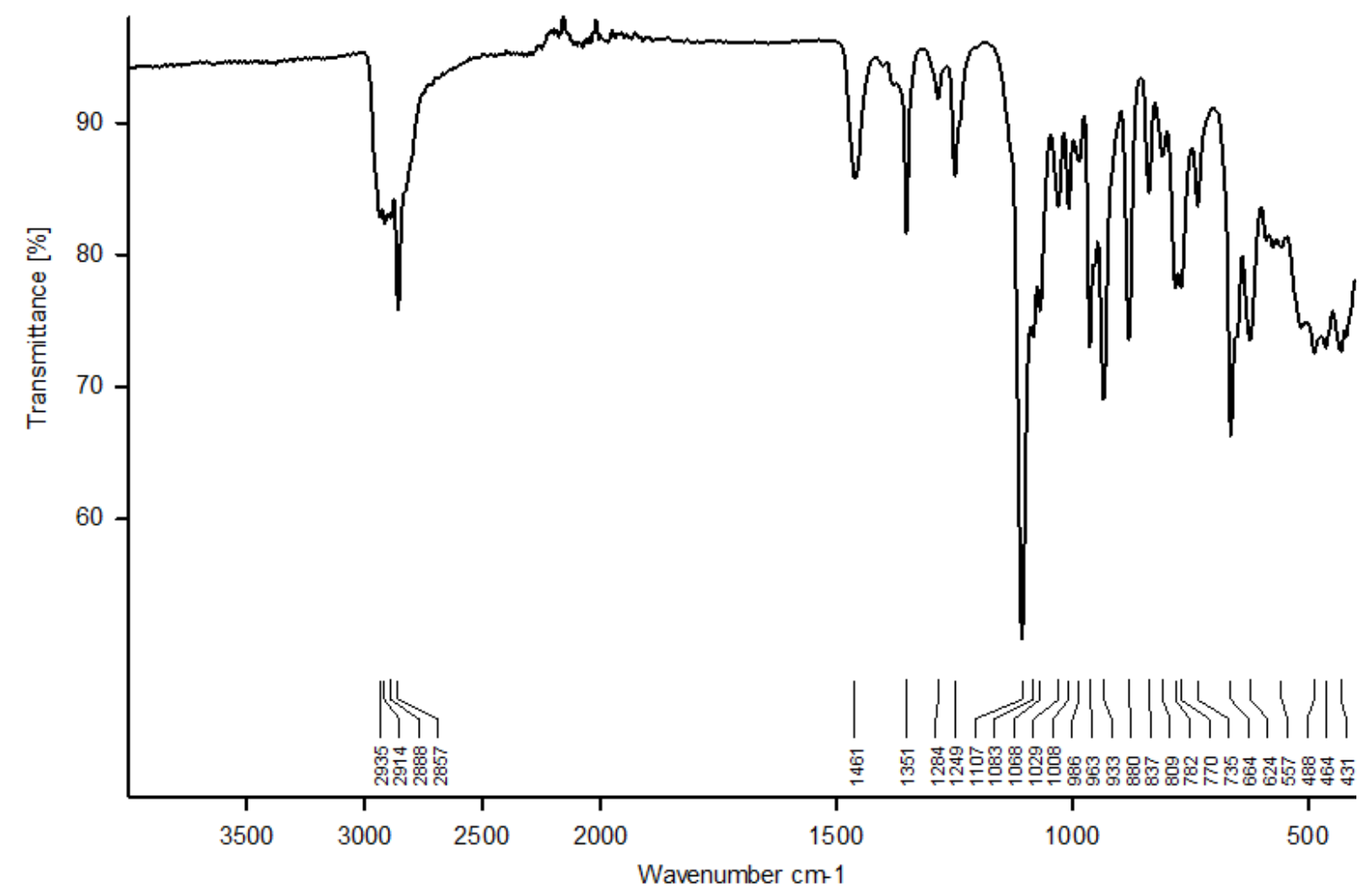

Figure S6. ATR-IR spectrum of 1-3.

4. Raman Spectroscopy

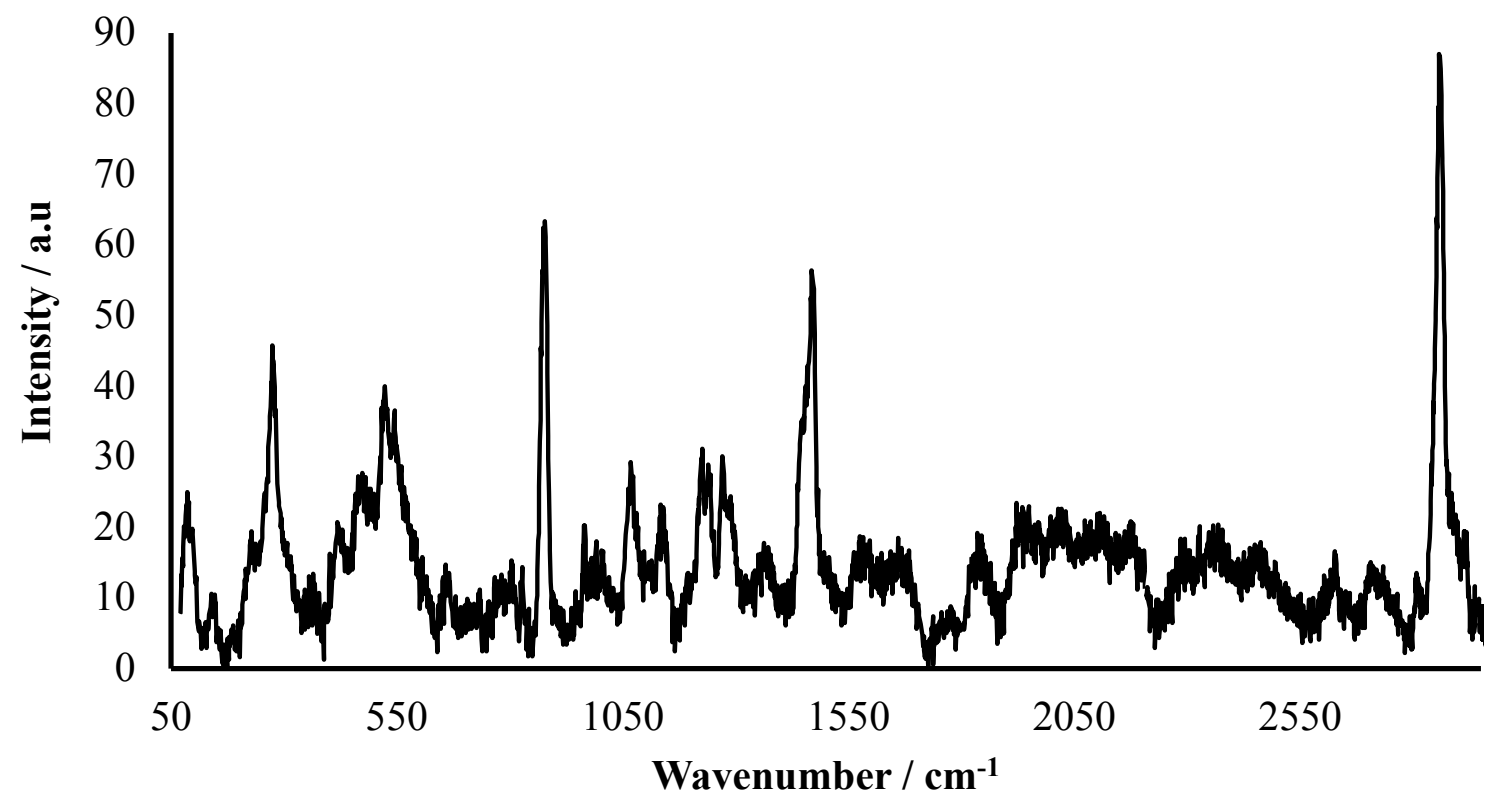

Figure S7. Raman spectrum of 1-3. 
5. UV/Vis Spectroscopy

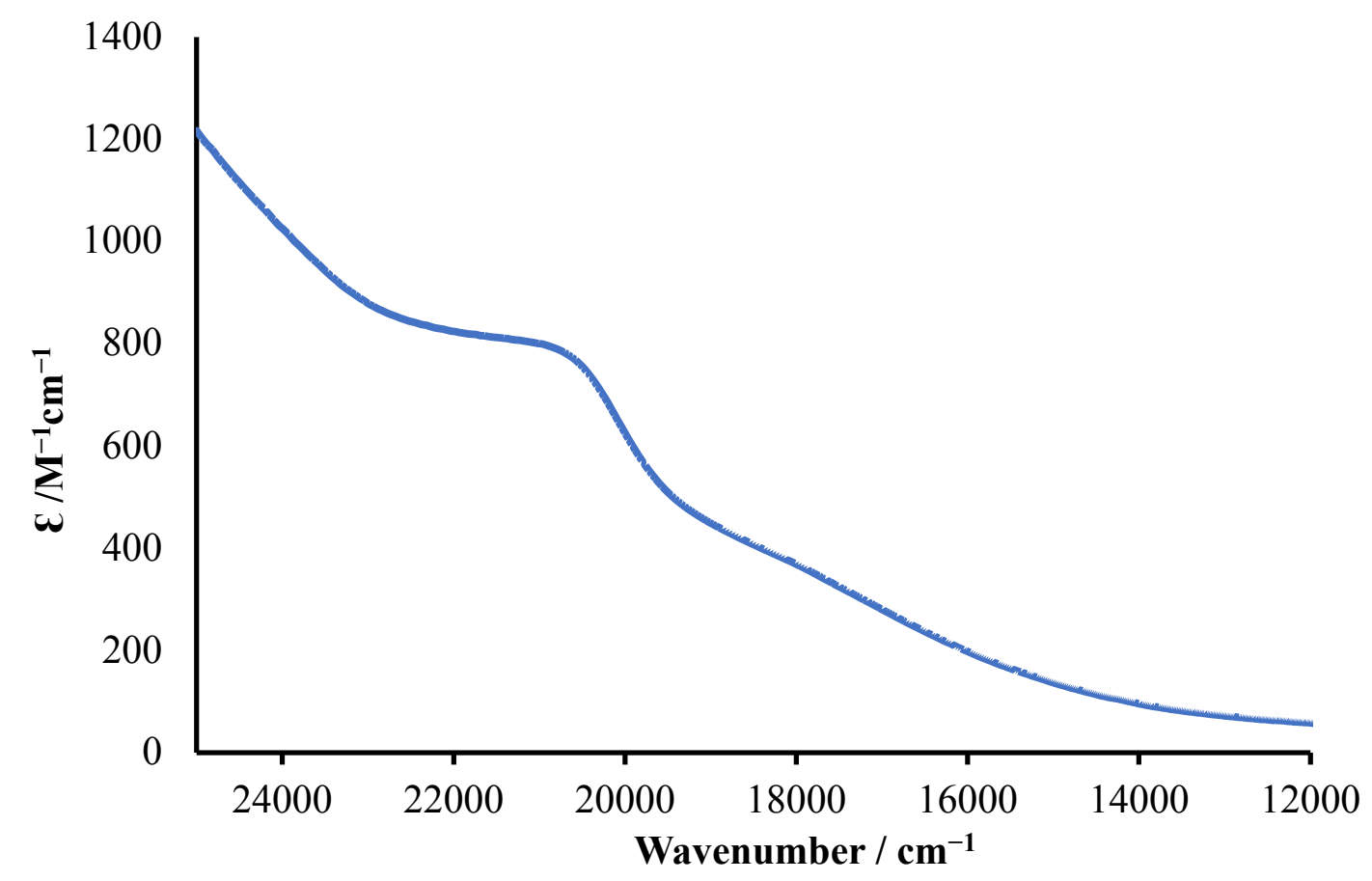

Figure S8. UV/Vis spectrum of 1-3. 


\section{Electrochemistry}

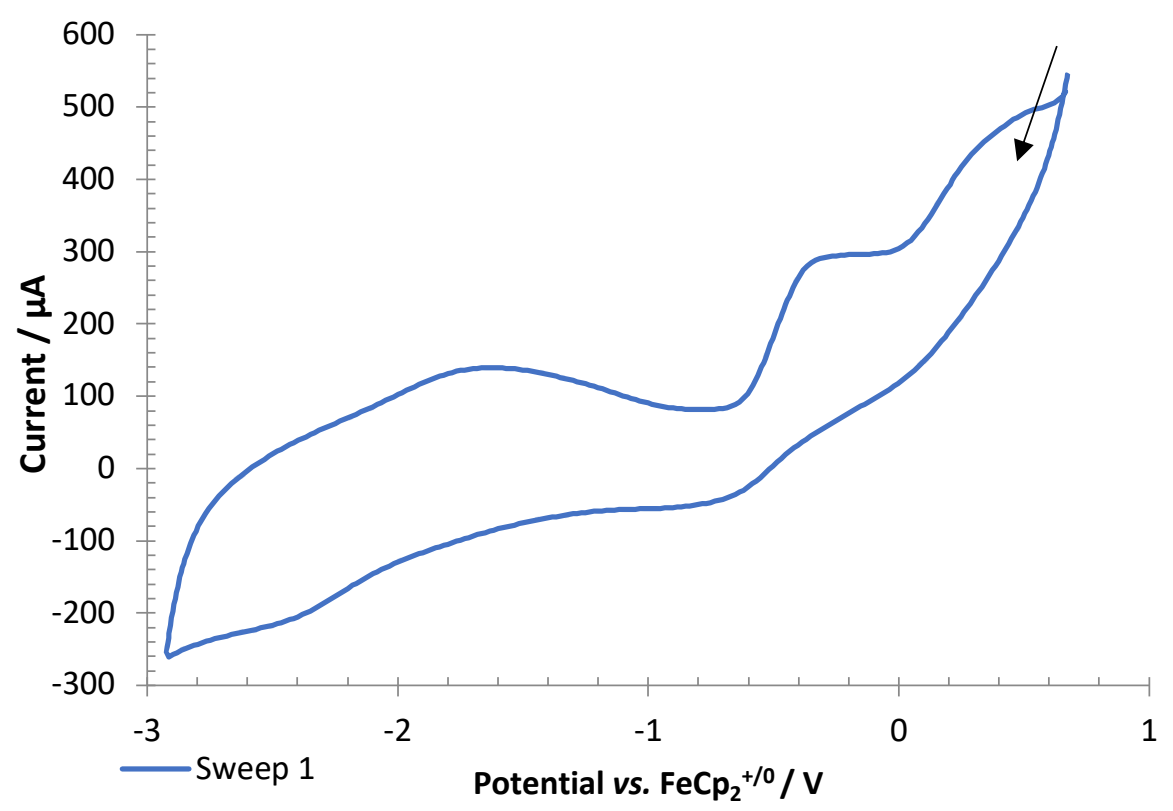

Figure S9. Voltammogram (1 cycle) for $1 \mathrm{mM}$ of a mixture of 1-3 in $0.5 \mathrm{M}$ $\left[\mathrm{Bu}_{4} \mathrm{~N}\right]\left[\mathrm{BF}_{4}\right] / \mathrm{DME}$, scan rate $500 \mathrm{mV} \mathrm{s}^{-1}$. The arrow indicates sweep direction.

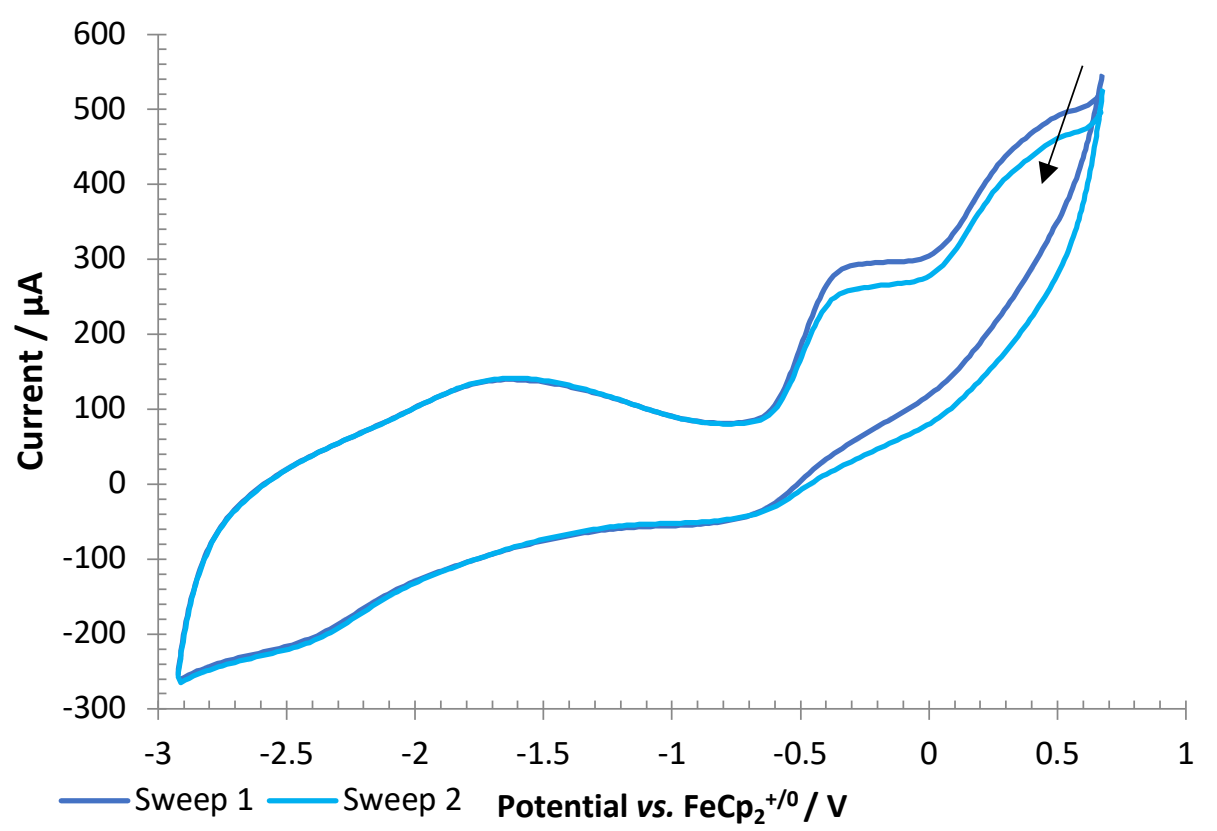

Figure S10. Voltammogram (2 cycles) for $1 \mathrm{mM}$ of a mixture of 1-3 in $0.5 \mathrm{M}$ $\left[\mathrm{Bu}_{4} \mathrm{~N}\right]\left[\mathrm{BF}_{4}\right] / \mathrm{DME}$, scan rate $500 \mathrm{mV} \mathrm{s}^{-1}$. The arrow indicates sweep direction. 


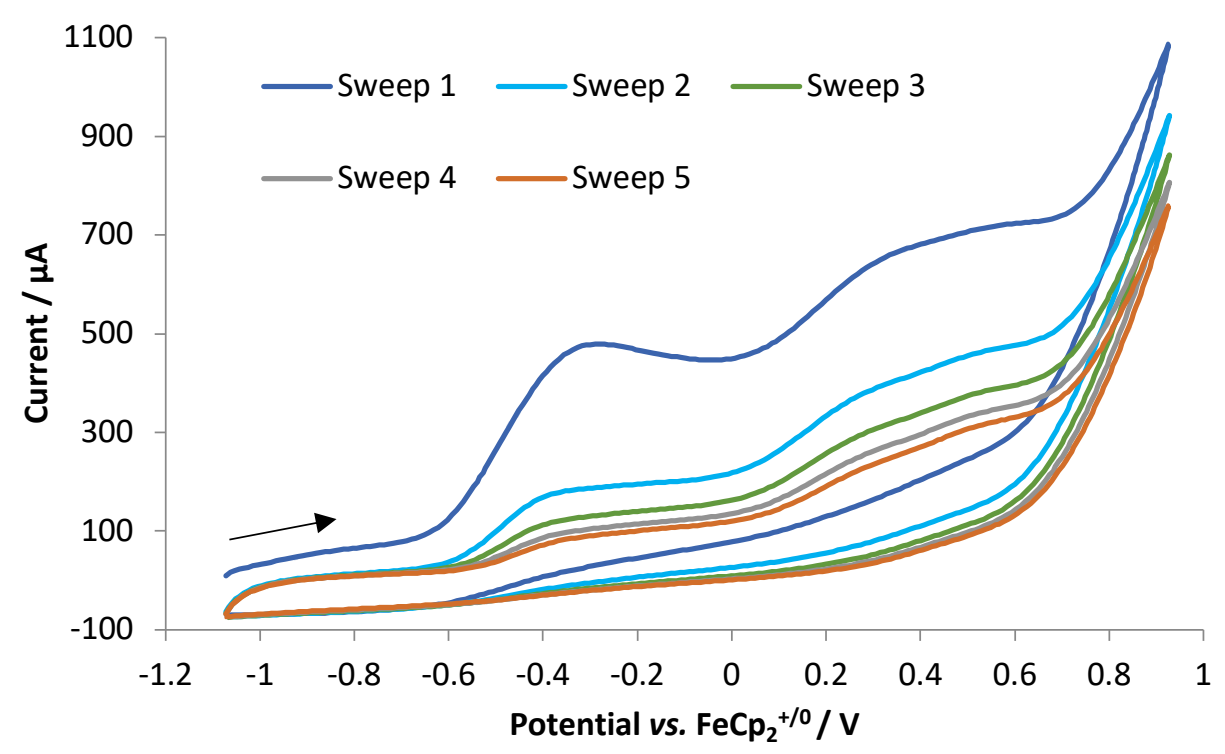

Figure S11. Voltammogram (5 cycles) for $1 \mathrm{mM}$ of a mixture of 1-3 in $0.5 \mathrm{M}$ $\left[\mathrm{Bu}_{4} \mathrm{~N}\right]\left[\mathrm{BF}_{4}\right] / \mathrm{DME}$, scan rate $500 \mathrm{mV} \mathrm{s}^{-1}$. The arrow indicates sweep direction. The zoomed in region of the potential $v s . \mathrm{FeCp}_{2}{ }^{+/ 0}$ between 1 and -1.2 better shows the decomposition of $\mathbf{1}$ after successive sweeps.

\section{Reduction Experiments}

\section{Attempted Reduction of 1}

To a solution of $1(25.0 \mathrm{mg}, 10.0 \mu \mathrm{mol})$ in $\mathrm{C}_{6} \mathrm{D}_{6}(0.3 \mathrm{~mL})$, a mixture of $\mathrm{KC}_{8}(2.00 \mathrm{mg}, 10.0$ $\mu \mathrm{mol})$ and 18-crown-6 $(5.00 \mathrm{mg}, 20.0 \mu \mathrm{mol})$ in $\mathrm{C}_{6} \mathrm{D}_{6}(0.1 \mathrm{~mL})$ was added. The mixture was agitated for 10 mins and then heated at $70{ }^{\circ} \mathrm{C}$ for $12 \mathrm{hrs} .{ }^{1} \mathrm{H}$ NMR and IR spectra were recorded of the resultant mixture of products. ${ }^{1} \mathrm{H}$ NMR $\left(\mathrm{C}_{6} \mathrm{D}_{6}, 298 \mathrm{~K}\right): 0.77\left(\mathrm{t},{ }^{3} \mathrm{~J}_{\mathrm{HH}}=6.1 \mathrm{~Hz}\right.$, $2 \mathrm{H}), 0.87\left(\mathrm{t},{ }^{3} \mathrm{~J}_{\mathrm{HH}}=7.3 \mathrm{~Hz}, 2 \mathrm{H}\right), 1.14(\mathrm{~s}, \mathrm{br}, 57 \mathrm{H}), 1.49(\mathrm{~s}, \mathrm{br}, 48 \mathrm{H}), 1.57(\mathrm{~s}, \mathrm{br}, 20 \mathrm{H}), 2.44(\mathrm{t}$, $\left.{ }^{3} \mathrm{~J}_{\mathrm{HH}}=6.1 \mathrm{~Hz}, 6 \mathrm{H}\right), 2.64(\mathrm{~s}, \mathrm{br}, 3 \mathrm{H}), 2.74(\mathrm{~s}, \mathrm{br}, 3 \mathrm{H}), 2.90\left(\mathrm{q},{ }^{3} \mathrm{~J}_{\mathrm{HH}}=6.1 \mathrm{~Hz}, 6 \mathrm{H}\right), 3.07(\mathrm{~s} \mathrm{br}$, 4H), $3.36(\mathrm{~s}, \mathrm{br}, 43 \mathrm{H}), 3.42(\mathrm{~s}, 9 \mathrm{H}), 3.44(\mathrm{~s}, 9 \mathrm{H}), 3.56(\mathrm{~s}$ br, $6 \mathrm{H}), 3.67$ (s br, 14H), 3.85 (s, $5 \mathrm{H}), 3.95\left(\mathrm{~d},{ }^{3} \mathrm{~J}_{\mathrm{HH}}=6.1 \mathrm{~Hz}, 3 \mathrm{H}\right), 4.13(\mathrm{~s}, 3 \mathrm{H}), 4.17(\mathrm{~s}, 2 \mathrm{H}), 6.42\left(\mathrm{q},{ }^{3} \mathrm{~J}_{\mathrm{HH}}=7.3 \mathrm{~Hz}, 1 \mathrm{H}\right)$; FTIR $v / \mathrm{cm}^{-1}$ (Neat): 3397 (w, br, NH), 2940 (s), 2863 (s), 2713 (w), 1881 (m br), 1615 (m) 1461 
(m), $1400(\mathrm{w}), 1352(\mathrm{~m}), 1247(\mathrm{~m}), 1201(\mathrm{~m}), 1104(\mathrm{~s}), 1012(\mathrm{w}), 985(\mathrm{w}), 960(\mathrm{w}), 881(\mathrm{~m})$, $829(\mathrm{w}), 751(\mathrm{w}), 695(\mathrm{w}), 631(\mathrm{w})$.

\section{Attempted Reduction of 1}

To a solution of $1(25.0 \mathrm{mg}, 10.0 \mu \mathrm{mol})$ in $\mathrm{C}_{6} \mathrm{D}_{6}(0.2 \mathrm{~mL})$, a mixture of $\mathrm{KC}_{8}(3.00 \mathrm{mg}, 10.0$ $\mu \mathrm{mol})$ and 18-crown-6 $(11.0 \mathrm{mg}, 40.0 \mu \mathrm{mol})$ in $\mathrm{C}_{6} \mathrm{D}_{6}(0.1 \mathrm{~mL})$ was added. The mixture was agitated for $10 \mathrm{mins}$ and heated at $70{ }^{\circ} \mathrm{C}$ for $12 \mathrm{hrs} .{ }^{1} \mathrm{H}$ NMR and IR spectra were recorded of the resultant mixture of products. ${ }^{1} \mathrm{H}$ NMR $\left(\mathrm{C}_{6} \mathrm{D}_{6}, 298 \mathrm{~K}\right): 0.77\left(\mathrm{t},{ }^{3} \mathrm{~J}_{\mathrm{HH}}=6.7 \mathrm{~Hz}, 2 \mathrm{H}\right), 0.87(\mathrm{t}$, $\left.{ }^{3} \mathrm{~J}_{\mathrm{HH}}=7.3 \mathrm{~Hz}, 1 \mathrm{H}\right), 1.14(\mathrm{~s}, \mathrm{br}, 60 \mathrm{H}), 1.49(\mathrm{~s}, \mathrm{br}, 58 \mathrm{H}), 1.57(\mathrm{~s}, \mathrm{br}, 27 \mathrm{H}), 2.44\left(\mathrm{t},{ }^{3} \mathrm{~J}_{\mathrm{HH}}=6.1\right.$ $\mathrm{Hz}, 6 \mathrm{H}), 2.65$ (s, br, 4H), $2.74(\mathrm{~s}, \mathrm{br}, 2 \mathrm{H}), 2.90\left(\mathrm{q},{ }^{3} \mathrm{~J}_{\mathrm{HH}}=6.7 \mathrm{~Hz}, 6 \mathrm{H}\right), 3.07$ (s, br, 4H), 3.40 (s, br, 55H), $3.42(\mathrm{~s}, 14 \mathrm{H}), 3.45(\mathrm{~s}, 18 \mathrm{H}), 3.56(\mathrm{~s}, \mathrm{br}, 6 \mathrm{H}), 3.67(\mathrm{~s}, \mathrm{br}, 13 \mathrm{H}), 3.85(\mathrm{~s}, 6 \mathrm{H}), 3.95$ $\left(\mathrm{d},{ }^{3} \mathrm{~J}_{\mathrm{HH}}=6.1 \mathrm{~Hz}, 3 \mathrm{H}\right), 4.13(\mathrm{~s}, 4 \mathrm{H}), 4.17(\mathrm{~s}, 1 \mathrm{H}), 6.42\left(\mathrm{q},{ }^{3} \mathrm{~J}_{\mathrm{HH}}=7.3 \mathrm{~Hz}, 1 \mathrm{H}\right) ;$ FTIR $v / \mathrm{cm}^{-1}$ (Neat): 3388 (w, br, NH), 2938 (m), 2863 (s), 2700 (w), 1612 (m), 1461 (m), 1352 (m), 1319 (w), $1288(\mathrm{w}), 1247(\mathrm{w}), 1202(\mathrm{~m}), 1103(\mathrm{~s}), 1012(\mathrm{w}), 984(\mathrm{~m}), 960(\mathrm{~m}), 881(\mathrm{w}), 836(\mathrm{w})$, $764(\mathrm{w}), 669(\mathrm{w}), 568(\mathrm{w})$.

\section{Computational Details}

Unrestricted geometry optimizations for $\mathbf{1}$ were performed using coordinates derived from the crystal structure of $\mathbf{1}$ as the starting point. During the geometry optimizations no symmetry was imposed on the model. A single point energy calculation was then performed with no symmetry restraint. Calculations were performed using the Amsterdam Density Functional (ADF) suite version 2012 with the standard convergence criteria. ${ }^{8,9}$ The DFT geometry optimizations employed Slater type orbital (STO) triple- $\zeta$-plus polarization allelectron basis sets (from the Dirac and ZORA/TZP database of the ADF suite). Scalar relativistic approaches (spin-orbit neglected) were used within the ZORA Hamiltonian ${ }^{10-12}$ for 
the inclusion of relativistic effects and the local density approximation (LDA) with the correlation potential due to Vosko et al was used in all of the calculations. ${ }^{13}$ Generalized gradient approximation corrections were performed using the functionals of Becke and Perdew. ${ }^{14,15}$ MOLEKEL ${ }^{16}$ was used to prepare the three-dimensional plots of the electron density. The final energy from a single point energy calculation on the geometry optimized coordinates of 1 was $-943.80082548 \mathrm{eV}$.

\section{References}

1. CrysAlisPRO version 39.46, Oxford Diffraction /Agilent Technologies UK Ltd, Yarnton, England.

2. Sheldrick, G.M. SHELXT - Integrated space-group and crystal-structure determination. Acta Cryst. Sect. A, 2015, 71, 3-8.

3. Sheldrick, G.M. Crystal structure refinement with SHELXL. Acta Cryst. Sect. C, 2015, 71, 3-8.

4. Dolomanov, O. V.; Bourhis, L. J.; Gildea, R. J.; Howard, J. A. K.; Puschmann, H. OLEX2: a complete structure solution, refinement and analysis program. J. Appl. Cryst., 2009, 42, 339-341.

5. Farugia, L. J. WinGX and ORTEP for Windows: an update. J. Appl. Cryst., 2012, 45, 849-854.

6. Farugia, L. J. WinGX and ORTEP for Windows: an update. J. Appl. Cryst., 2012, 45, 849-854.

7. Persistence of Vision (TM) Raytracer: Persistence of Vision Pty. Ltd., Williamstown, Victoria, Australia.

8. Fonseca Guerra, C.; Snijders, J. G.; Te Velde, G.; Baerends, E. J. Towards an order-N 
DFT Method. Theor. Chem. Acc., 1998, 99, 391-403.

9. Te Velde, G.; Bickelhaupt, F. M.; Van Gisbergen, S. J.; Fonseca Guerra, A. C.; Baerends, E. J.; Snijders, J. G.; Ziegler, T. Chemistry with ADF. J. Comput. Chem., 2001, 22, 931967.

10. Van Lenthe, E.; Baerends, E. J.; Snijders, J. G. Relativistic regular two-component Hamiltonians. J. Chem. Phys., 1993, 99, 4597-4610.

11. Van Lenthe, E.; Baerends, E. J.; Snijders, J. G. Relativistic total energy using regular approximations. J. Chem. Phys., 1994, 101, 9783-9792.

12. Van Lenthe, E.; Ehlers, A. E.; Baerends, E. J. Geometry optimization in the Zero Order Regular Approximation for relativistic effects. J. Chem. Phys., 1999, 110, 8943-8953.

13. Vosko, S. H.; Wilk, L.; Nusair, M. Accurate spin-dependent electron liquid correlation energies for local spin density calculations: a critical analysis. Can. J. Phys., 1980, 58, 1200-1211.

14. Becke, A. D. Density-functional exchange-energy approximation with correct asymptotic behaviour. Phys. Rev. A., 1988, 38, 3098.

15. Perdew, J. P. Density-functional approximation for the correlation energy of the inhomogeneous electron gas. Phys. Rev. B., 1986, 33, 8822.

16. Portmann, S.; Luthi, H. P. MOLEKEL: An interactive molecular graphics tool. Chimia, 2000, 54, 766-770. 